\title{
„Politischer Kurs für mehr gesundheitliche Vorsorge bestätigt"
}

DFZ: In anderen Freien Berufen wurden die Honorare immer wieder den wirtschaftlichen Entwicklungen angepasst. Bei den Zahnärzten ist das seit 27 Jahren nicht passiert. Nun soll wieder einmal ein neuer Datensatz erhoben werden. Sie drücken sich doch nur vor der Entscheidung einer Anhebung?

Heidenblut: Wir schließen uns der Einschätzung der Bundesregierung in dem Bericht an, dass kein dringender Handlungsbedarf für eine Änderung der GOZ besteht.

Als besonders erfreuliches Ergebnis des Berichtes verbuche ich die Verschiebung der Umsatzanteile zugunsten der prophylaktischen und konservierenden Leistungen. Das bestätigt die Politik in ihrem Kurs für mehr gesundheitliche Vorsorge und die Zahnärzteschaft in ihrer Vorreiterrolle in der Präventionsarbeit.

Um in Zukunft auf eine noch differenziertere und aussagekräftigere Datengrundlage zurückgreifen zu können, plant das Bundesministerium für Gesundheit eine Studie. Sie soll herausfinden, wo Anpassungen der Datengrundlage notwendig sind. Der Beobachtungszeitraum wird voraussichtlich bis 2017 ausgedehnt.

DFZ: Selbst die Bundesregierung erkennt inzwischen an, dass die Kosten für Material und Praxis erheblich gestiegen sind. Warum sind in die derzeit gültige GOZ keine aktuellen wissenschaftlichen Erkenntnisse und zahnmedizinischen Entwicklungen eingeflossen? Heidenblut:Im Gegenteil, ein erklärtes Hauptziel für die Erarbeitung der GOZ 2012 war die Anpassung an das aktuelle Versorgungsgeschehen. Die Zahnärzteschaft wurde durch Fachgesellschaften hinzugezogen. Die Material- und Laborkosten stehen nicht in Verbindung mit Veränderungen der GOZ, denn sie werden zum Beispiel bei Gold für Zahnersatz an den Versicherten weitergegeben. Sie sind deswegen gesondert von dem Kostenanteil für zahnärztliche Leistungen zu betrachten. Der Bericht stellt außerdem fest, dass das privatzahnärztliche Honorar, abzüglich der Material- und Laborkosten, um 9,2 Prozent gestiegen ist.

\section{Hier steht eine Anzeige.}

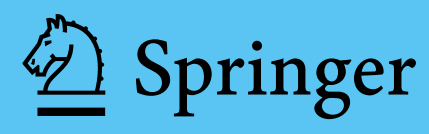

